\title{
Substituting steel for a polymer in a jar for ball milling does matter
}

\author{
Evgeniy Losev*a,b, Sergey Arkhipov ${ }^{a, b}$, Dmitry Kolybalov ${ }^{b}$, Alexey Mineev ${ }^{a, b}$, Andrey Ogienko $^{a, c}$, \\ Elena Boldyreva*a,b, Vladimir Boldyrev ${ }^{b, d}$ \\ ${ }^{a}$ Boreskov Institute of Catalysis SB RAS, Lavrentiev avenue, 5, Novosibirsk, 630090 Russia \\ ${ }^{b}$ Novosibirsk State University, Pirogova street, 2, Novosibirsk, 630090 Russia \\ 'LLC "SP IIC" Moscow-Novosibirsk, Russia \\ ${ }^{d}$ Voevodsky Institute of Chemical Kinetics and Combustion SB RAS, Novosibirsk, 630090 Russia \\ contact: eboldyreva@yahoo.com
}

\begin{abstract}
Usually, in situ diffraction studies of mechanochemical transformations use plastic milling jars in place of steel. This is done to reduce the absorbtion of radiation by the walls. Using as an example the polymorphic transformation of $\beta$-glycine, we show that the transformation rate can vary significantly depending on the material of the jars. Using ex situ analysis we here compare the transformation rates in steel and common plastics such as acrylonitrile butadiene styrene, polylactic acid, and polyethylene terephthalate glycol.
\end{abstract}

Already hundreds of syntheses achieved by mechanical action on powder mixtures have been documented [1]. Mechanochemical reactions do not require the use of solvents and are often highly selective with yields approaching $100 \%$. Mechanical action can also induce polymorphic transformations or amorphisation. Following transformations in situ, directly in the milling jar during mechanical treatment, is highly demanded. In situ experiments allow to monitor the progress of the transformations without interrupting the process. Such studies are becoming increasingly frequent. Diffraction experiments are carried out at synchrotron radiation sources [2]. The instruments for the mechanical processing (most often - ball mills) that are installed at the stations at synchrotron radiation facilities are rather similar to the commercially available mills, or are designed specially [3-5]. To make the milling jar more transparent for X-ray radiation, the steel jar (typically used in a laboratory or in industry) is substituted for one made of an organic polymer, often polymethylmethacrylate (PMMA), or sometimes polyimide. Plastic jars can be 3D-printed, and this new technology becomes more accessible even for small laboratories [4,5]. The choice of the material for 3D-printed jars looks rather arbitrary. Additives of polymers to the sample are well-known to have a pronounced effect on the outcome of mechanochemical transformations: they can stabilize the amorphous states and metastable polymorphs within a mechanocomposite "polymer - organic compound" [6-9], or facilitate the synthesis of cocrystals of organic compounds (POLAG, polymer assisted grinding) $[10,11]$. One can expect that even substituting the metal jar for a polymer jar can have a significant effect on the outcome of the mechanical treatment. Sample-jar interactions are different for steel and plastics because of the difference in mechanical properties, thermoconductivity, surface charge, hydrophilic or hydrophobic properties and many other characteristics. Conversely, metals can act as catalysts for organic reactions [12]. The results obtained with a plastic jar in a model device used in synchrotron experiments may not be therefore directly indicative of the same process conducted in a metal jar in the laboratory. This makes it difficult to compare correctly the results of ex situ and in situ mechanochemical experiments, as well as of the in situ experiments performed by different groups.

The aim of this work was to compare the results of mechanical treatment of a sample from the same batch in the same mill and following exactly the same protocol with the only difference being the jar material: steel or different polymers (polylactic acid PLA, Polyethylene terephthalate glycol PETG, and acrylonitrile butadiene styrene $A B S$ ). For a case study we took a sample of the $\beta$-polymorph of glycine $\left({ }^{+} \mathrm{NH}_{3}-\mathrm{CH}_{2}-\mathrm{COO}-\right)$ prepared as fine powder.

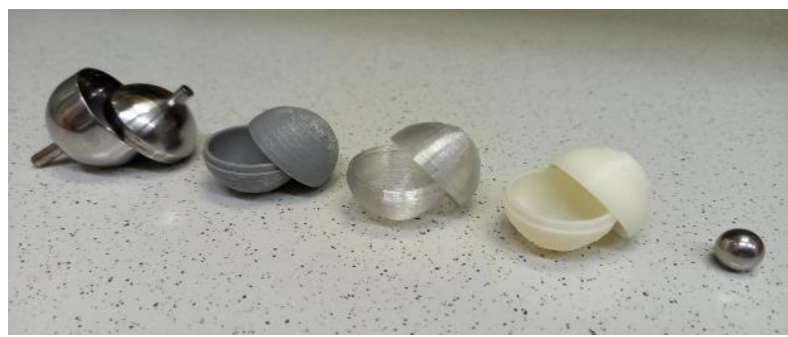

Figure 1. The steel jar of the vibrational mill Vibrator DDR-GM9458 NARVA $50 \mathrm{HZ}$, and 3D-printed polymer inserts into it (from left to right: PLA, PETG and $A B S$ ), as well as a steel ball (9.5 mm diameter, $3.5 \mathrm{~g}$ )

$\beta$-Glycine was prepared by freeze-drying from a $5 \%$ solution in a mixed THF-water solvent containing 19.6 mass \% of THF) [13]. The light porous sample had density of $\sim 50 \mathrm{mg} / \mathrm{cm}^{3}$ ), with a narrow particle size distribution, with particles $<1 \mu \mathrm{m}$ diameter. Between the experiments, samples of $\beta$-glycine were stored in closed containers in a desiccator over annealed silica gel (relative humidity about $20 \%$ ) to exclude any interaction with the moisture from the air. Before and after each experiment the samples were characterised by X-ray powder diffraction at a laboratory diffractometer (STOE STADI MP, CuK $\alpha_{1}, \lambda=1.54060$ $\mathrm{A}^{\circ}, 40 \mathrm{kV} / 40 \mathrm{~mA}$, bent $\mathrm{Ge}$ (111) monochromator, Dectris MYTHEN $1 \mathrm{~K}$ linear detector). The results were analysed using WinXPOW 3.05 software [Stoe \& Cie (2009). WinXPOW. Version 3.05. Stoe \& Cie, Darmstadt, Germany]. The samples were mechanically treated in a vibrational mill Vibrator DDR-GM9458 NARVA $(50 \mathrm{~Hz}$, a single steel ball with $9.5 \mathrm{~mm}$ diameter and 
mass $3.5 \mathrm{~g})$. The treatment was continuous during 20 minutes. A standard sample mass was $15 \mathrm{mg}$. Polymer (PLA, PETG and $A B S$ ) inserts into the steel jar were 3D-printed using a Wanhao GR2 printer with a brass nozzle $(0.4 \mathrm{~mm}$ diameter) (Fig. 1). A glass mirror $(210 \times 250 \times 4 \mathrm{~mm})$ was used as a support. No significant heating of the milling jars was observed to result from treatment.

On storage or on heating, $\beta$-glycine has been reported to transform into the pure $\alpha$-polymorph, pure $\gamma$-polymorph, or a mixture of the two [14]. We have found that the mechanical treatment of $\beta$-glycine results in its polymorphic transformation into the pure $\alpha$-form. Remarkably, this is not the thermodynamically stable polymorph under ambient conditions. In the course of experiments, we have observed that the polymorphic transformation of the $\beta$-polymorph into $\alpha$ form was significantly faster if the ball was taken by the fingers before placing it into the jar. The powder sample stuck to the ball in this case, and this made mechanical action on it more efficient when a ball hit the jar wall (Fig. 2). We have then carried out two series of experiments: 1 . Experiments without control of the purity of the surfaces of the ball and the jar; 2 . Experiments in which the surfaces of the ball and the jar were carefully cleaned from even traces of grease from the fingers using soap foam with subsequent multiple rinsing by distilled water and drying in a special drying box. The surface of the ball was treated by ethanol and dried, and subsequently handled using a specially treated instrument.

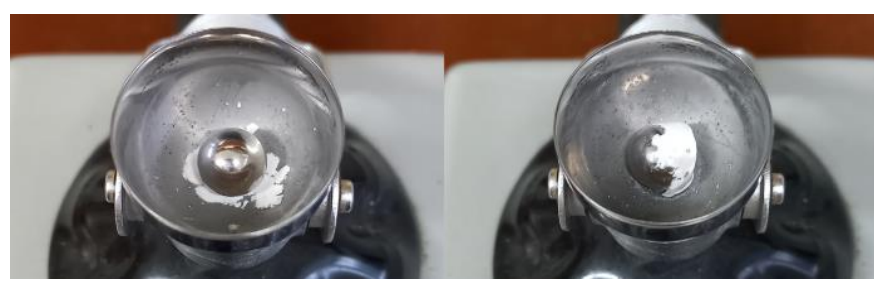

Figure 2. The effect of the finger traces at the ball surface on its interaction with the powder of $\beta$-glycine before the start of the experiment: left - the ball with the surface specially treated with ethanol and dried, put into the jar using a tweezer, right - the ball without a special treatment, put into the jar by fingers

Independent of the preliminary treatment of the ball and the jar, the $\alpha$-polymorph accumulated faster on mechanical treatment of the $\beta$-polymorph in a steel jar, as compared to the treatment when the polymer inserts were used (Fig. 3). This is contrary to the effect of polymers when added as powder and can be supposed to account for different rheology and not to chemical interactions between glycine and the polymers. For different types of polymer the transformation rate was similar for the jars made of PLA and PETG. No transformation into the $\alpha$-polymorph could be observed in the jars made of ABS (at least during the equivalent time).

For the jar made from the same material the degree of transformation of the $\beta$-polymorph into the $\alpha$-form was higher if the surface was not cleaned specially from fingers tracers and/or the ball was put into the jar by fingers (for the same treatment time). In the steel jars the transformation of the $\beta$ - polymorph into the $\alpha$ - polymorph was complete after 20 minutes of continuous treatment if the ball was specially rolled between the fingers before the experiment (Fig. 4). This effect of preliminary touching the ball with fingers seems to be related to the improved interaction of the $\beta$-glycine powder with the surface of the ball if traces of grease are present, improving the transfer of mechanical energy to the sample when the ball (with the sample on it) collides with the jar wall. The effect can be supposed to be more important when milling soft organic compounds in relatively low-energy devices, as compared with high-energy treatment of inorganic materials: if an inorganic sample is harder than steel, it will scratch the milling jars, so that the initial state of the surface will be no longer important. Besides, the higher milling energies in case of inorganic mechanochemistry usually give rise to higher local temperatures when grease - if initially available - will burn or evaporate. The possibility that chemical compounds present in the grease from the fingers can have a catalytic effect on the polymorphic transformation of the $\beta$ - to the $\alpha$-polymorph cannot also be excluded at this stage. More studies with other compounds are needed, to make unambiguous conclusions.

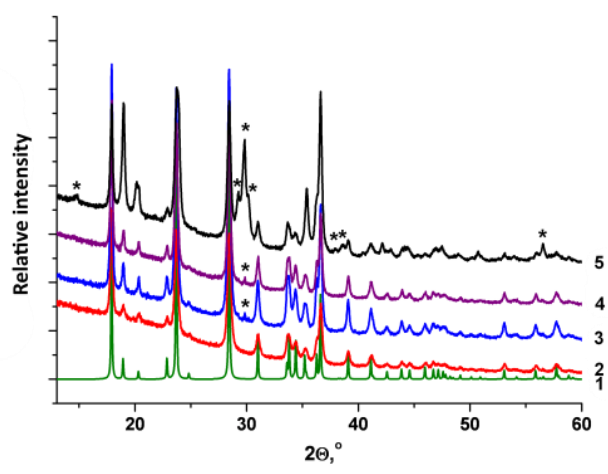

Figure 3. Powder X-ray diffraction patterns of the $\beta$-glycine mechanically treated continuously during 20 minutes in the jars made of different materials (experiments with specially cleaned surfaces of the ball and the jar). 1 - $\beta$-glycine (diffraction pattern calculated from single-crystal data); 2 - ABS jar (coincides with the pattern for the $\beta$-glycine before treatment); 3 -PETG jar; 4 - PLA jar; 5 - steel jar. Stars * mark the peaks of $\alpha$-glycine

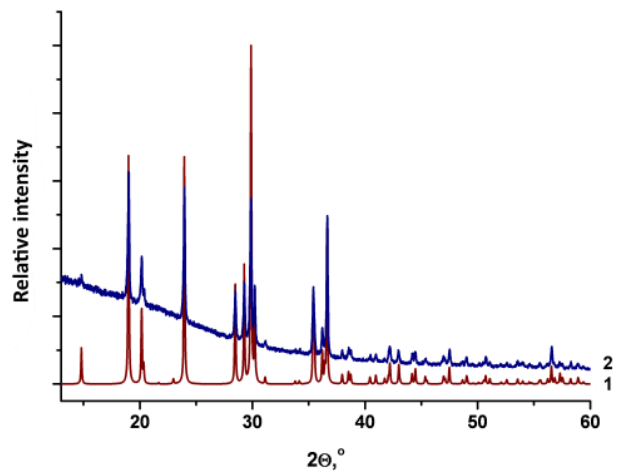

Figure 4. Powder X-ray diffraction pattern of $\beta$-glycine mechanically treated during 20 minutes with a ball that was deliberately rolled between the fingers before the start of the treatment. $1-\alpha$-glycine 
(pattern calculated from single-crystal data); 2 - $\beta$-glycine completely transformed into $\alpha$-glycine after the treatment in the steel jar
14 E. Boldyreva, Israel J. Chem. 2021 doi:10.1002/ijch.202100103

Thus, our case study has shown that a substitution of the steel jar for a polymer can reduce drastically the rate of the mechanochemical transformation in a vibrational ball mill. Also the choice of the polymer matters. This must be taken into account when planning in situ synchrotron radiation diffraction experiments. The transformation rate both in metal and in plastic jars may be increased, if the milling ball is preliminary touched by fingers and in this way greased, to improve the contact between the sample and the milling bodies.

This research was supported by Russian Foundation of Basic Research, project 19-29-12026-MK (EAL, SGA, AMM, EVB). Equipment of the REC MDEST of the Novosibirsk State University was used.

Evgeniy Losev - Data curation (performed experiments), Formal Analysis, Investigation, Methodology, Writing - original draft;

Sergey Arkhipov, Dmitry Kolybalov, Alexey Mineev - Data curation (designed and manufactured plastic vials);

Andrey Ogienko - Data curation (prepared and characterized the sample of the metastable $\beta$-glycine);

Elena Boldyreva - Conceptualization, Methodology, Writing original draft, review \& editing, Resources, Project administration

Vladimir Boldyrev - Conceptualization

There are no conflicts of interest to declare.

\section{Notes and references}

1 J. Andersen, J. Mack, Green Chemistry, 2018, 20(7), 1435

2 A.A. Michalchuk, A. Kabelitz and F. Emmerling, In Nontraditional Activation Methods in Green and Sustainable Applications. 2021, 369-419. Elsevier

3 T. Friščić, I. Halasz, P.J. Beldon, A.M. Belenguer, F. Adams, S.A. Kimber, V. Honkimäki and R.E. Dinnebier, Nature Chem., 2013, 5, 66

4 N.A. Tumanov, V. Ban, A. Poulain and Y. Filinchuk, J. Appl. Cryst., 2017, 50, 994

5 G.I. Lampronti, A.A.L. Michalchuk, P.P. Mazzeo, A. Belenguer J.K. Sanders, A. Bacchi and F. Emmerling, Nature Commun. 2021, 12(1). doi:10.1038/s41467-021-26264-1

6 T.P. Shakhtshneider, Solid State lonics, 1997, 101, 851

7 V.V. Boldyrev, J. Mater. Sci. 2004, 39(16), 5117

8 I. Colombo, G. Grassi and M. Grassi, J. Pharm. Sci. 2009, 98(11), 396133

9 P.N. Balani, S.Y. Wong, W.K. Ng, E. Widjaja, R.B.H. Tan, and S.Y. Chan, Intern. J. Pharm. 2010, 391(1-2), 125

10 D. Hasa, E. Carlino and W. Jones, Cryst. Growth \& Design, 2016, 16(3), 1772

11 L.S. Germann, S.T. Emmerling, M. Wilke, R.E. Dinnebier, M. Moneghini and D. Hasa, Chem. Commun. 2020, 56(62), 8743

12 A.A. Michalchuk, E.V. Boldyreva, A. Belenguer, F. Emmerling \& V.V. Boldyrev, Frontiers in Chemistry, 2021, 9, 359

13 A.G. Ogienko, V.A. Drebushchak, E.G. Bogdanova, A.S. Yunoshev, A.A. Ogienko, E.V. Boldyreva and A.Y. Manakov, J. Therm. Analys. Calorim., 2017, 127(2), 1593 